\title{
Article
}

\section{Role of an additional interfacial spin- transfer torque for current-driven skyrmion dynamics in chiral magnetic layers}

Mackinnon, Callum Robert, Lepadatu, Serban, Mercer, Tim and Bissell, Philip

Available at https://clok.uclan.ac.uk/35557/

Mackinnon, Callum Robert orcid iconORCID: 0000-0001-5584-2545, Lepadatu, Serban orcid iconORCID: 0000-0001-6221-9727, Mercer, Tim orcid iconORCID: 0000-0002-1557-2138 and Bissell, Philip orcid iconORCID: 0000-0002-80241757 (2020) Role of an additional interfacial spin-transfer torque for currentdriven skyrmion dynamics in chiral magnetic layers. Physical Review B (PRB), 102 (21). p. 214408. ISSN 2469-9950

It is advisable to refer to the publisher's version if you intend to cite from the work. http://dx.doi.org/10.1103/PhysRevB.102.214408

For more information about UCLan's research in this area go to http://www.uclan.ac.uk/researchgroups/ and search for <name of research Group>.

For information about Research generally at UCLan please go to http://www.uclan.ac.uk/research/

All outputs in CLoK are protected by Intellectual Property Rights law, including Copyright law. Copyright, IPR and Moral Rights for the works on this site are retained by the individual authors and/or other copyright owners. Terms and conditions for use of this material are defined in the policies page. 


\title{
Role of an additional interfacial spin-transfer torque for current-driven skyrmion dynamics in chiral magnetic layers
}

\author{
Callum Robert MacKinnon $\odot,{ }^{*}$ Serban Lepadatu $\odot,{ }^{\dagger}$ Tim Mercer $\odot,{ }^{\ddagger}$ and Philip Raymond Bissell $\odot^{\S}$ \\ Jeremiah Horrocks Institute for Mathematics, Physics and Astronomy, University of Central Lancashire, Preston PR1 2HE, United Kingdom
}

(Received 8 September 2020; revised 12 November 2020; accepted 12 November 2020; published 7 December 2020)

\begin{abstract}
Skyrmions can be driven by spin-orbit torques as a result of the spin Hall effect. Here we model an additional contribution in ultrathin multilayers, arising from the spin accumulation at heavy metal/ferromagnetic interfaces and observe the effects on a large range of skyrmion diameters. The combination of the interfacial spin-transfer torque and the spin-orbit torque results in skyrmion motion which helps to explain the observation of small skyrmion Hall angles for skyrmion diameters less than $100 \mathrm{~nm}$. We show that this additional term has a significant effect on the skyrmion dynamics and leads to rapidly decreasing skyrmion Hall angles for small skyrmion diameters, as well as a skyrmion Hall angle versus skyrmion velocity dependence nearly independent of the surface roughness characteristics. Also, the effect of various disordered energy landscapes, in the form of surface roughness, on the skyrmion Hall angle and velocity is shown to be largely drive dependent. Our results show good agreement with those found in experiments thus concluding that the interfacial spin-transfer torque should be included in micromagnetics simulations for the reproduction of experimental results.
\end{abstract}

DOI: $10.1103 /$ PhysRevB.102.214408

\section{INTRODUCTION}

Skyrmions are topologically protected quasiparticlelike magnetic structures [1], which are of great interest as they can be efficiently manipulated using spin torques due to applied currents in ultrathin magnetic multilayers, thus opening the possibility of a new class of spintronics devices [2-4]. Skyrmions have been observed at room temperature in systems with broken inversion symmetry [5-9], stabilized by the interfacial Dzyaloshinskii-Moriya interaction (DMI) [10,11], and more recently current-induced skyrmion motion has been demonstrated in ultrathin magnetic multilayers [12-19] by utilizing spin currents due to the spin Hall effect (SHE) which gives rise to spin-orbit torques (SOTs) [20-24]. The SHE governs SOT via the accumulation of spins at the boundaries of a current-carrying nonmagnetic heavy metal (NM)/ferromagnetic (FM) bilayer due to the generation of transverse spin currents. The SHE exploits the spin-orbit coupling in a NM to convert the unpolarized charge into a pure spin current which arises from both intrinsic and extrinsic effects and gives rise to an asymmetric scattering of conduction electrons. Irrespective of the underlying origins of the SHE and thus spin accumulation at the NM/FM interface, the SOT exerted on the FM magnetization can be broken down into two components, namely, the dampinglike (DL) SOT and the fieldlike (FL) SOT.

Current-driven skyrmion motion is currently hindered by the skyrmion Hall angle (SkHA), $\theta_{\mathrm{SkHA}}$, in which the

\footnotetext{
*CRMackinnon@uclan.ac.uk

†SLepadatu@uclan.ac.uk

†TMercer1@uclan.ac.uk

§PBissell@uclan.ac.uk
}

skyrmion path deviates from the direction of the applied charge current [18], which poses problems for spintronics applications. Furthermore, the SkHA has been shown to vary with the applied current strength [15-19], and this effect is not reproduced by analytical or numerical models in ideal structures. While material imperfections play a very important role [25], there are many gaps in the literature, and a full understanding of how the SkHA varies with driving strength, skyrmion diameter, skyrmion chirality, and varying levels of magnetic and nonmagnetic imperfections is required. In recent experimental work, a SkHA largely independent of skyrmion diameter has been observed [14]; however, analytical results based on the Thiele equation predict a significant increase in SkHA with decreasing skyrmion diameter, and this discrepancy is significantly pronounced for small diameters below $100 \mathrm{~nm}$. In another study [18] experimental results were also compared to modeling based on SOT for very large skyrmions with diameters above $800 \mathrm{~nm}$. Experimental results and SOT simulations show good agreement for large skyrmion diameters, which showed slightly larger SkHA values for the SOT model; however, the SOT model predictions deviate significantly from the experimental results at small skyrmion diameters [14] even when very large damping values are assumed. Reducing the SkHA is critical for spintronic devices and it has been theoretically shown that the spin-orbit coupling in a NM/FM bilayer can be used to tune the skyrmion Hall effect by controlling gate voltages to steer skyrmions [26], and furthermore adjusting the strength of the Rashba spin-orbit coupling has been show to result in a negligible SkHA [27].

Very recently, it was proposed that interfacial spin-transfer torques (ISTTs) [28] may also be important, and thus a more complete model based on both SOT and ISTT can be formed. Here we show, using micromagnetics modeling coupled with 
a self-consistent spin transport solver in multilayers, the addition of ISTT results in a significantly reduced discrepancy between experimental and modeling results, and good agreement is obtained with recent experimental results which have demonstrated a nearly diameter-independent SkHA [14]. In this paper, we study the effect of the additional ISTT term on skyrmion motion in a Co $(1 \mathrm{~nm}) / \mathrm{Pt}(3 \mathrm{~nm})$ bilayer with and without surface disorder in the form of surface roughness as a function of skyrmion diameter. An overview of the spin transport model is given in Sec. II. In Sec. III we study the disorder-free case, investigating skyrmion motion as a function of skyrmion diameter and interfacial spin mixing conductance. Finally, in Sec. IV we also consider the effect of surface roughness on skyrmion motion, where the SkHA is shown to vary with current density as the skyrmion motion changes from pinned, to creep, and finally plastic flow regime. The results obtained for a range of skyrmion diameters are shown to collapse onto a universal SkHA versus skyrmion velocity dependence, nearly independent of the surface roughness characteristics.

\section{SPIN TRANSPORT MODEL}

The effect of spin torques on skyrmion motion is modeled using the Landau-Lifshitz-Gilbert (LLG) equation:

$$
\frac{\partial \mathbf{m}}{\partial t}=-\gamma \mathbf{m} \times \mathbf{H}_{\mathrm{eff}}+\alpha \mathbf{m} \times \frac{\partial \mathbf{m}}{\partial t}+\frac{1}{M_{S}} \mathbf{T}_{\mathbf{S}} .
$$

Here $\gamma=\mu_{0} g_{\text {rel }}\left|\gamma_{e}\right|$, where $\gamma_{e}$ is the electron gyromagnetic ratio, $g_{\text {rel }}$ is a relative gyromagnetic factor, $\alpha$ is the Gilbert damping, and $M_{s}$ is the saturation magnetization. $\mathbf{H}_{\text {eff }}$ is the effective field term consisting of several additive terms, namely, the demagnetizing field, exchange interaction including the interfacial Dzyaloshinskii-Moriya exchange interaction in the $x y$ plane, applied magnetic field, and uniaxial magnetocrystalline anisotropy. $\mathbf{T}_{s}$ is the total spin-torque term, which includes contributions from SOT and ISTT, and can be computed self-consistently using the drift-diffusion model where the spin current density is given as

$$
\mathbf{J}_{S}=-\frac{\mu_{B}}{e} P \sigma \mathbf{E} \otimes \mathbf{m}-D_{e} \nabla \mathbf{S}+\theta_{\mathrm{SHA}} \frac{\mu_{B}}{e} \boldsymbol{\varepsilon} \sigma \mathbf{E} .
$$

Here, the drift-diffusion model shows three distinct components, where $\mathbf{J}_{S}$ is the spin current such that $\mathbf{J}_{S i j}$ indicates the flow of the $j$ component of spin polarization in the $i$ direction. The first is the contribution due to drift included in the FM layers, where $P$ is the current spin-polarization, $\sigma$ is the electrical conductivity, $\mathbf{m}$ is the normalized magnetization, and $\mathbf{E}$ is the electric field. The second is a diffusive term, where $D_{e}$ is the electron diffusion constant and $\mathbf{S}$ is the spin accumulation. The third term is the SHE in the NM layers, where $\theta_{\text {SHA }}$ is the spin Hall angle and $\boldsymbol{\varepsilon}$ is the rank-3-unit asymmetric tensor. The drift-diffusion model also includes the charge current density, given as $\mathbf{J}_{C}=\sigma \mathbf{E}$, which is simplified here by assuming the inverse SHE is negligible.

Transverse components of spin currents are absorbed at a NM/FM interface resulting in a spin torque which can be included in the drift-diffusion model with the incorporation of the spin mixing conductance, $G^{\uparrow \downarrow}$, with the resulting total spin torque obtained using circuit theory boundary conditions
[29] as

$$
\begin{aligned}
\mathbf{T}_{S}= & \frac{g \mu_{B}}{e d_{F}}\left[\operatorname{Re}\left\{G^{\uparrow \downarrow}\right\} \mathbf{m} \times\left(\mathbf{m} \times \Delta \mathbf{V}_{S}\right)\right. \\
& \left.+\operatorname{Im}\left\{G^{\uparrow \downarrow}\right\} \mathbf{m} \times \Delta \mathbf{V}_{S}\right] .
\end{aligned}
$$

Here $d_{F}$ is the ferromagnetic layer thickness; $\Delta \mathbf{V}_{S}$ is the spin chemical potential drop across a NM/FM interface, where $\mathbf{V}_{S}=\left(D_{e} / \sigma\right)\left(e / \mu_{B}\right) \mathbf{S}$. The drift-diffusion model can be solved analytically to obtain the following expression for the SOT, given in Eq. (4), by assuming negligible in-plane spin diffusion.

$$
\mathbf{T}_{\text {SOT }}=\theta_{\text {SHAeff }} \frac{\mu_{B}}{e} \frac{\left|J_{c}\right|}{d_{F}}\left[\mathbf{m} \times(\mathbf{m} \times \mathbf{p})+r_{G} \mathbf{m} \times \mathbf{p}\right] .
$$

The SOT contains both dampinglike and fieldlike components, with $r_{G}$ being the FL-SOT coefficient. Here $\mathbf{p}=$ $\mathbf{z} \times \mathbf{e}_{J c}$, where $\mathbf{e}_{J c}$ is the charge current direction. The quantity $\theta_{\text {SHAeff }}$ is the effective spin Hall angle, proportional to the real, or intrinsic, spin Hall angle, $\theta_{\mathrm{SHA}} ; \theta_{\mathrm{SHAeff}}$ and $r_{G}$ are given in Eqs. (5) and (6), respectively.

$$
\begin{aligned}
\theta_{\text {SHAeff }}= & \theta_{\text {SHA }}\left(1-\frac{1}{\cosh \left(d_{N} / \lambda_{s f}^{N}\right)}\right) \\
& \times \frac{\operatorname{Re}\{\tilde{G}\}^{2}-\operatorname{Im}\{\tilde{G}\}^{2}+N_{\lambda} \operatorname{Re}\{\tilde{G}\}}{\left(N_{\lambda}+\operatorname{Re}\{\tilde{G}\}\right)^{2}+\operatorname{Im}\{\tilde{G}\}^{2}}, \\
r_{G}= & \frac{N_{\lambda} \operatorname{Im}\{\tilde{G}\}+2 \operatorname{Re}\{\tilde{G}\} \operatorname{Im}\{\tilde{G}\}}{N_{\lambda} \operatorname{Re}\{\tilde{G}\}+\operatorname{Re}\{\tilde{G}\}^{2}-\operatorname{Im}\{\tilde{G}\}^{2}} .
\end{aligned}
$$

Here $\quad N_{\lambda}=\tanh \left(d_{N} / \lambda_{s f}^{N}\right) / \lambda_{s f}^{N}$ and $\tilde{G}=2 G^{\uparrow \downarrow} / \sigma_{N} . \quad$ As shown previously [30], it is important to make the distinction between the intrinsic spin Hall angle, and the effective spin Hall angle at the NM/FM interface, as the latter can be significantly smaller depending on the interface transparency as modeled using the spin mixing conductance.

Another important source of vertical spin current is due to the NM/FM interlayer diffusion of a spin accumulation generated in the FM layer, resulting in an interfacial spin-torque contribution. In ultrathin films the interlayer spin diffusion results in spin torques similar in form to the bulk Zhang-Li STT $[31,32]$, but greatly enhanced partly due to the inverse dependence on $d_{F}$ and acting in the opposite direction. This additional interfacial spin torque is given in Eq. (7).

$$
\mathbf{T}_{\text {ISTT }}=-\left[\left(\mathbf{u}_{\perp} \cdot \nabla\right) \mathbf{M}-\frac{\beta_{\perp}}{M_{S}} \mathbf{M} \times\left(\mathbf{u}_{\perp} \cdot \nabla\right) \mathbf{M}\right] .
$$

The interfacial spin torque acts in the opposite direction to the Zhang-Li STT, and the spin-drift velocity and the nonadiabaticity parameter are replaced by an effective perpendicular spin-drift velocity, Eq. (8), and effective nonadiabaticity parameter.

$$
\mathbf{u}_{\perp}=\mathbf{J}_{C} \frac{\left|P_{\perp}\right| g \mu_{B}}{2 e M_{S}} \frac{1}{1+\beta_{\perp}^{2}} .
$$

Here $P_{\perp}$ is an effective perpendicular spin polarization parameter, which in the case of ISTT takes on negative values. These values are not dependent on a single material alone but depend on the transport properties of both the NM and FM layers [28]. 
The ISTT arises due to an imbalance in the spin accumulation either side of the NM/FM interface. This imbalance gives rise to diffusive vertical spin currents, which contain both longitudinal and transverse spin components. The transverse spin components are absorbed at the interface, and due to conservation of total angular momentum this results in an interfacial spin torque. The ISTT thus arises due to absorption of transverse spin components, as does the SOT, but in this case the vertical spin currents originate in the FM layer, not the NM layer. The spin imbalance arises due to the spin accumulation generated at magnetization gradients and is due to the nonzero divergence of the in-plane spin current in the FM layer, as well as the spin mistracking effect due to the exchange rotation and spin dephasing processes. This is the same transverse spin accumulation which gives rise to the Zhang-Li adiabatic and nonadiabatic spin-transfer torques; however, the ISTT arises due to absorption of vertical, transverse spin components at the interface, not due to the in-plane, transverse spin absorption lengths, and is thus an interfacial spin torque with strength inversely dependent on the FM layer thickness. We note that experimental evidence for a huge negative spin-transfer torque was obtained for ultrathin Co layers interfaced with Pt [33]. This torque was found to act along the current direction with a negative spin polarization, scaling inversely to the Co layer thickness, and for ultrathin Co layers its strength far exceeds that of the bulk STT and is comparable to or greater than the SOT, and thus similar to the ISTT torque in Eq. (7) discussed here. Our results could provide an explanation for these observations; however, the analysis of this case is left for future work.

Experimental results are often compared to an analytical model known as the Thiele equation [34], which can be used to describe the dynamics of magnetic skyrmions due to various driving forces. There is an included caveat in assuming skyrmions have rigid body magnetic characteristics; however, this allows the skyrmions to be considered with steady-state velocity arising from the equilibrium between different forces acting on the rigid body. The Thiele equation includes (i) the force acting on the skyrmion; in the bilayer systems, this total force results from the DL SOT and ISTT; (ii) the skyrmion Hall effect, whereby the skyrmion motion deviates from the charge current direction due to the Magnus force [35]; (iii) the dissipative force, which includes a dissipative tensor and the Gilbert damping coefficient. The solution to the Thiele equation is shown in Eq. (9), which gives an expression for the diameter-dependent and drive-independent SkHA [15,36].

$$
\begin{aligned}
v & =\sqrt{\frac{\left(u_{\mathrm{SOT}}-\beta D u_{\mathrm{STT}}\right)^{2}+u_{\mathrm{STT}}^{2}}{1+(\alpha D)^{2}},} \\
\tan \left(\theta_{\mathrm{SkHA}}\right) & =\frac{u_{\mathrm{SOT}}+(\alpha-\beta) D u_{\mathrm{STT}}}{\alpha D u_{\mathrm{SOT}}-\left(1+\alpha \beta D^{2}\right) u_{\mathrm{STT}}} .
\end{aligned}
$$

Here $D=R / 2 \Delta$, where $\Delta$ is the domain wall width and $R$ is the skyrmion radius. Moreover $u_{\mathrm{SOT}}=$ $\mu_{B} \theta_{\mathrm{SHAeff}} \pi R J_{\mathrm{NM}} / 4 e M_{S} d_{F}$, and $u_{\mathrm{STT}}=P g \mu_{B} J_{\mathrm{FM}} / 2 e M_{S}(1+$ $\beta^{2}$ ), where $J_{\mathrm{NM}}$ and $J_{\mathrm{FM}}$ are the current densities in the NM and FM layers, respectively. Equation (9) models the contribution of both the SOT and ISTT for current-driven skyrmion motion and is in agreement with micromagnetics modeling for the ideal skyrmion case as we have verified.

\section{SPIN TORQUES IN A DISORDER-FREE SYSTEM}

Current-induced Néel skyrmion movement has been observed in asymmetric stacks including $[\mathrm{Pt} / \mathrm{Co} / \mathrm{Ta}]_{x}$ [12], $\mathrm{Pt} / \mathrm{GdFeCo} / \mathrm{MgO}$ [13], $\mathrm{Ta} / \mathrm{CoFeB} / \mathrm{TaO}_{x}$ [37], and $\mathrm{Pt} / \mathrm{Co} / \mathrm{Ir}$ [17] as well as more complex stacks such as $\mathrm{Ta} / \mathrm{Pt} /[\mathrm{Pt} / \mathrm{CoB} / \mathrm{Ir}]_{x} \mathrm{Pt}$ [14] and symmetric stacks [38]. To study the effect of the SOT and ISTT on skyrmion motion, a $\operatorname{Pt}(3 \mathrm{~nm}) / \mathrm{Co}(1 \mathrm{~nm})$ bilayer thin film $[39,40]$ structure was chosen for this work, using periodic boundary conditions for the demagnetizing field. The current was applied across the bilayer through electrodes attached on its $x$-axis ends.

Figure 1(a) illustrates the SOT and ISTT components acting on a current-driven skyrmion with the combined effect displayed, which is a vector addition of the two torques. To investigate the effect of spin torques on an isolated skyrmion, first, a disorder-free bilayer was used, neglecting thermal fluctuations and material imperfections, with a fixed current density. The skyrmion diameter is controlled using an out-ofplane magnetic field in the $+z$ direction ranging from $\left|H_{z}\right|=$ $0.875 \mathrm{kA} \mathrm{m}^{-1}$ to $\left|H_{z}\right|=54 \mathrm{kA} \mathrm{m}^{-1}$, which yielded diameters ranging from 396 to $21 \mathrm{~nm}$, respectively. The full dependence of the skyrmion diameter on the applied field is shown in Appendix A. The results for the ideal case are shown in Fig. 2. For the spin torques obtained with the self-consistent spin transport solver, we show two distinct contributions and the combined torque effect. The contributions observed are the SOT due to SHE, as well as another important contribution obtained due to the interlayer diffusion of spin currents, namely, the ISTT. To obtain solutions for these contributions, $P_{\perp}, \beta_{\perp}, r_{G}$, and $\theta_{\text {SHAeff }}$ were obtained numerically. Thus the spin accumulation was computed using the spin transport solver, and the total spin torque obtained from Eq. (3). Then, Eqs. (4) and (7) for the SOT and ISTT, respectively, were used to obtain the above constants as fitting parameters to the total spin torque. While $\theta_{\mathrm{SHAeff}}$ and $r_{G}$ do not depend on the skyrmion diameter, $P_{\perp}$ and $\beta_{\perp}$ are not constant and show a small dependence on skyrmion diameter due to spin diffusion, similar to the enhancement of Zhang-Li nonadiabaticity obtained for vortex cores [41,42]. The dependence of these parameters on skyrmion diameter is shown in Appendix B. Figure 2(a) shows three clear distinct curves which are identified by the dashed, solid, and dash-dot lines which represent the SOT, the full spin torque, and ISTT, respectively. There is a clear difference in each line suggesting each component has a significant contribution to the skyrmion motion. The ISTT has a significant effect on the SkHA which is only applicable for ultrathin films due to the inverse thickness dependence, and NM layers with a small spin diffusion length, such as the $\mathrm{Co} / \mathrm{Pt}$ bilayer investigated here. Figure 2(b) shows the skyrmion path for each component, which shows that the SOT has the largest effect on the skyrmion motion compared to the ISTT component, as can be deduced from the SOT and ISTT skyrmion displacements. For large diameters the SOT and full torque dependencies tend towards each other and become near identical. This is an important result as it shows the SOT-modeled results for the skyrmion motion to be a good assumption for large diameters. However, for small skyrmion 


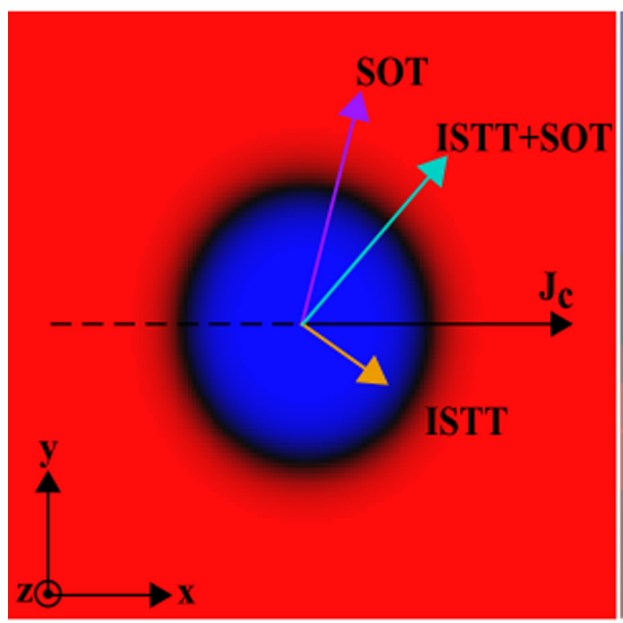

(a)

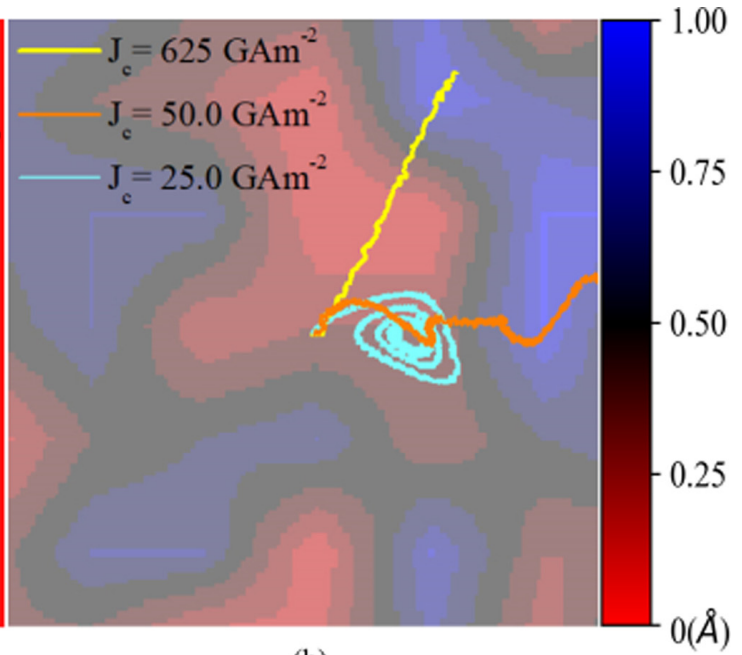

(b)

FIG. 1. (a) A snapshot of a 400-nm-diameter Néel skyrmion, showing the $z$ magnetization component. The magenta, orange, and cyan arrows represent the direction in which the SOT, ISTT, and the combination of these two torques in the spin transport solver act on the skyrmion when a current is applied, respectively. The black arrow indicates the conventional current direction, $\mathbf{J}_{\mathbf{c}}$. (b) Skyrmion motion in a bilayer system displaying pinned, creep, and flow regimes in cyan, orange, and yellow, respectively. The contoured effect outlines the disordered pattern on the surface of the sample with depth displayed by the heat map. All paths were obtained using a damping constant $\alpha=0.03$ and disorder variation period of $60 \mathrm{~nm}$.

diameters, the two trends deviate rapidly, which would explain discrepancies between experimental and theoretical results. The combined torque results correspond well with results obtained experimentally both for small skyrmion diameters [14] as well as for large diameters [14,15,18].

We note the ISTT is not confined to Néel type skyrmions alone as the ISTT arises from spin accumulation at magneti- zation gradients and is therefore applicable for any magnetic texture including antiskyrmions, skyrmioniums, and domain walls; however, investigation of these cases is outside the scope of this work.

The imaginary part of the spin mixing conductance, $\operatorname{Im}\left(G^{\uparrow \downarrow}\right)$, is commonly neglected with an effective spin mixing conductance used instead. The imaginary component is
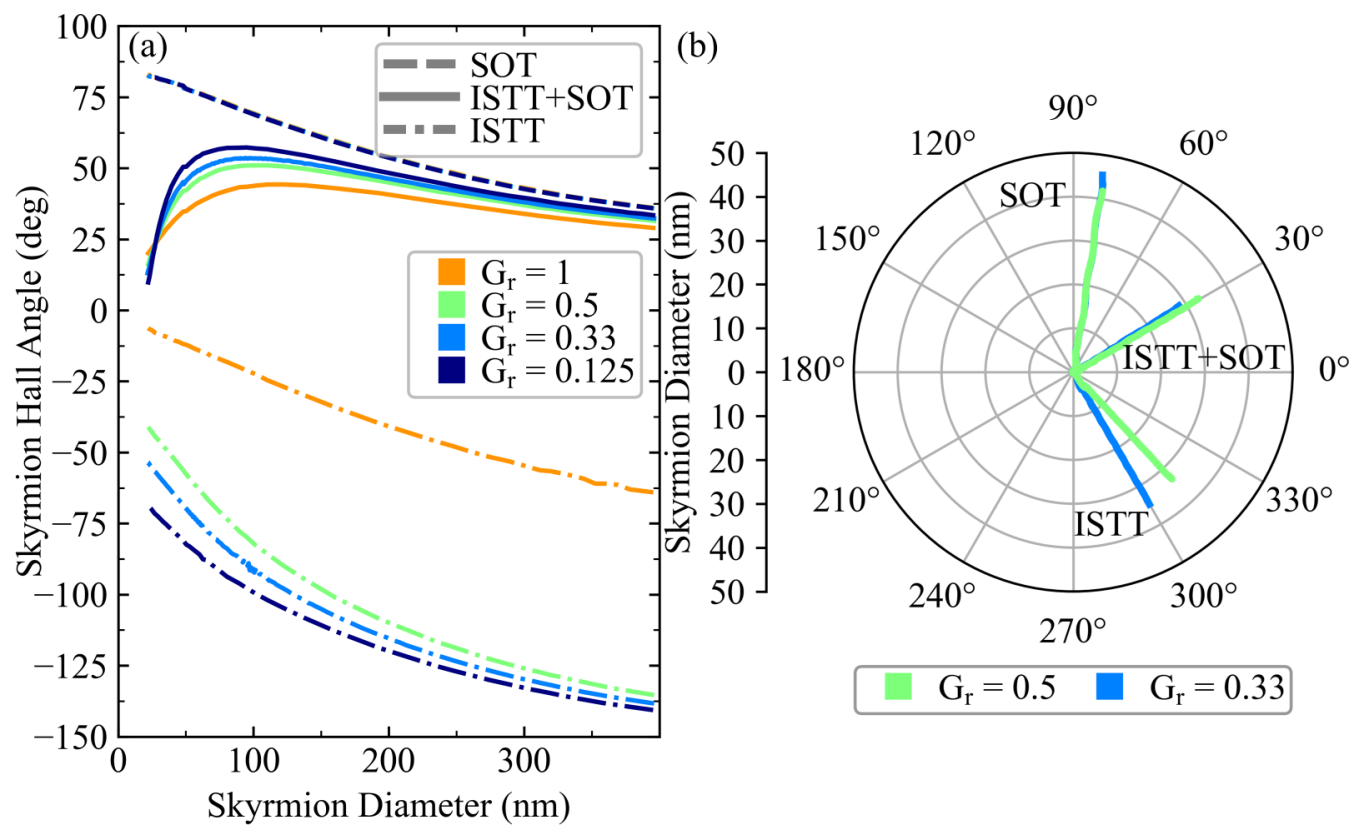

FIG. 2. Relationship between the skyrmion Hall angle and skyrmion diameter for different imaginary to real spin mixing conductance ratios, $G_{r}$. (a) Individual SOT and ISTT contributions for each ratio represented by a dashed and dash-dot line, respectively, as well as the full spin transport solver solution including both SOT and ISTT shown by the solid lines. (b) Skyrmion paths for $G_{r}=0.33$ and 0.5 . The individual SOT and ISTT contributions are shown alongside the full spin transport solver path. The current is applied along the $x$ direction, $0^{\circ}$. To obtain these results a damping value of $\alpha=0.1$ was used. 
usually assumed to be much smaller than the real component; however, imaginary to real spin mixing conductance ratios, $G_{r}=\operatorname{Im}\left(G^{\uparrow \downarrow}\right) / \operatorname{Re}\left(G^{\uparrow \downarrow}\right)$, larger than 1 have been measured recently [43]. Figure 2(a) shows the dependence of SkHA on the spin mixing conductance ratio with a clear influence from the ratio apparent. The SOT is not affected by $G_{r}$ in terms of the SkHA, but skyrmion velocity is affected by the $G_{r}$ ratio, as is apparent in Fig. 2(b) in which the velocity is larger for $G_{r}=0.33$. Furthermore, the $G_{r}$ ratio affects the ISTT contribution of both velocity and SkHA. The peaks of the combined torque curves range from $44^{\circ}$ to $57.3^{\circ}$ for $G_{r}=1$ and $G_{r}=0.125$, respectively. Figure 2(b) shows the skyrmion paths for each component for $G_{r}=0.33$ and 0.5 , illustrating further the velocity dependence on $G_{r}$. The ratio used in all subsequent modeling throughout this paper is determined using both DL- and FL-SOT components, values of which are given in Ref. [19] for a Pt/Co $(0.9 \mathrm{~nm})$ bilayer, which are used to obtain the fieldlike torque coefficient. The imaginary component of the spin mixing conductance can then be determined numerically by rearranging Eq. (6). From this we obtain $\operatorname{Im}\left(G^{\uparrow \downarrow}\right)=4.49 \times 10^{14} \mathrm{~S} \mathrm{~m}^{-2}$ and hence $G_{r}=0.3$.

In this work we concentrated on a single NM/FM interface, modeled by a single spin mixing conductance. It should be noted that there is a possibility for another contribution due to back reflection of spins from the second FM surface, particularly in NM/FM/NM multilayers when the FM layer thickness is comparable to or lower than the spin dephasing length. One approach to modeling the effect of the second surface is to use the concept of transmitted spin mixing conductance [44]. As shown in this reference this results in modified SOT strength, but the model now requires an additional spin mixing conductance. Moreover the presence of an additional NM layer will further enhance the ISTT and investigation of such structures is left for future work. However, while the exact quantitative dependence of the skyrmion Hall angle on skyrmion diameter is expected to change, qualitatively the conclusions are unaffected, namely, the ISTT generated at magnetization gradients, such as skyrmions, in multilayers needs to be taken into consideration in addition to the SOT.

Reference [19] reported on the current-driven motion of Néel skyrmions with diameters of the order of $100 \mathrm{~nm}$ in a $\mathrm{Pt} / \mathrm{Co} / \mathrm{MgO}$ trilayer, with and without disorder. Micromagnetics simulations were carried out to validate experimental results and the drive dependence of the skyrmion Hall effect was not observed to be accounted for by the FL SOT. An analytical model is shown for damping coefficients $\alpha=0.43,0.30$ and shows relatively good agreement to the experimental results. It was suggested that a recreation of the SkHA could be achieved using the description of SOT only with the use of large damping factors, whereas if a smaller damping factor was used the result would be significantly different due to the model relation given by Eq. (9) when $u_{\mathrm{STT}}=0$. It should be noted that there is disagreement in the literature for damping values for ultrathin Co films, with a range of values reported using different methods [45-52], including time-resolved magnetooptical Kerr effect (TRMOKE), ferromagnetic resonance (FMR), tight-binding modeling (TB), and domain wall (DW) motion. The damping generally increases with decreasing film thickness, which is due to a combination of increased effect of spin-orbit coupling and magnon-electron scattering at interfaces. Moreover, the presence of a heavy metal layer, such as $\mathrm{Pt}$, further increases the damping values due to spin pumping. Large values of damping have been reported, obtained indirectly from DW motion experiments, namely, $\alpha=0.43$ for $\mathrm{Pt}(3 \mathrm{~nm}) / \mathrm{Co}(0.9 \mathrm{~nm})$ [19], and $\alpha=0.3$ for $\operatorname{Pt}(4.5 \mathrm{~nm}) / \operatorname{Co}(0.8$ $\mathrm{nm}) / \mathrm{Pt}(3.5 \mathrm{~nm})$ [52] in the precessional DW motion regime, although when the steady DW motion regime was used values ranging up to $\alpha=3.4$ were reported [52]. On the other hand, direct measurement techniques have shown much smaller values for comparable systems, typically of the order of $\alpha=$ 0.1 or below. Thus FMR measurements obtain $\alpha=0.03$ for $\mathrm{Pt}(1.5 \mathrm{~nm}) / \mathrm{Cu}(10 \mathrm{~nm}) / \mathrm{Co}(1 \mathrm{~nm}) / \mathrm{Cu}(10 \mathrm{~nm})[46], \alpha=0.08$ for $\mathrm{Pt}(1.5 \mathrm{~nm}) / \mathrm{Co}(1 \mathrm{~nm}) / \mathrm{W}(1.5 \mathrm{~nm})[46], \alpha=0.02$ for $\operatorname{Co}(1.4$ $\mathrm{nm}) / \operatorname{Pt}(1 \mathrm{~nm})$ [45], and $\alpha=0.18$ for $\operatorname{Pt}(5 \mathrm{~nm}) / \operatorname{Co}(1 \mathrm{~nm}) / \operatorname{Pt}(2$ $\mathrm{nm})$ [51]. Similarly results from TRMOKE measurements obtain values of $\alpha=0.13$ for $[\mathrm{Co}(0.4 \mathrm{~nm}) / \operatorname{Pt}(0.8 \mathrm{~nm})]_{12}[50]$, $\alpha=0.2$ for $\operatorname{Pt}(5 \mathrm{~nm}) / \operatorname{Co}(1 \mathrm{~nm}) / \operatorname{Pt}(2 \mathrm{~nm})$ [51], and $\alpha=0.03$ extrapolated for $\mathrm{Pt}(4 \mathrm{~nm}) / \mathrm{Co}(1 \mathrm{~nm}) / \mathrm{AlO}_{x}$. TB modeling on $\mathrm{Co}(1 \mathrm{~nm}) / \operatorname{Pt}(1.2 \mathrm{~nm})$ also obtains a comparable value of $\alpha=$ 0.08 [48].

It is well known that for skyrmions an additional contribution arises due to the emergent magnetic field and emergent time-dependent electric field, resulting in a topological Hall effect (THE) and charge pumping effect, respectively $[42,53,54]$. As has been shown previously this results in an enhancement in the nonadiabaticity of vortex structures and skyrmions [42,53]. In the case of ISTT, however, the effective nonadiabaticity is strongly dependent on the FM layer thickness and the NM layer spin diffusion length, and for the $\mathrm{Co} / \mathrm{Pt}$ bilayer considered here $\left|\beta_{\perp}\right|>1$ as shown in Appendix B. As we have verified using an extended drift-diffusion model which includes the emergent electromagnetic field (for model implementation details see [55]), the effect on the skyrmion motion in the $\mathrm{Co} / \mathrm{Pt}$ bilayers studied here is negligible; however, further investigations are required to characterize the different possible regimes, including combinations of ISTT and bulk STT together with the THE and charge pumping, particularly as a function of FM layer thickness and NM layer transport properties.

\section{SKYRMION CHARACTERISTICS ON DISORDERED SURFACES}

In-depth theoretical investigations of skyrmions interacting with defects have shown significant changes to the skyrmion paths $[18,25,28,56-60]$ with further investigations showing disorder to have a large effect on the SkHA and skyrmion velocity $[14,16,19,28,38]$. There is also an inherent problem when using the Thiele equation for comparison due to the inability to incorporate skyrmion malleability. Furthermore, we investigate the effect surface disorder has on the SkHA with the ISTT component included in the simulations. This was accomplished by using a similar method to that used for the disorder-free results; however, in this case, a surface disorder is applied which is accomplished using a jagged surface roughness profile [28]. To investigate the effect of disorder on skyrmion motion, three surface roughness variation periods were applied, with 20, 40, and $60 \mathrm{~nm}$ average 


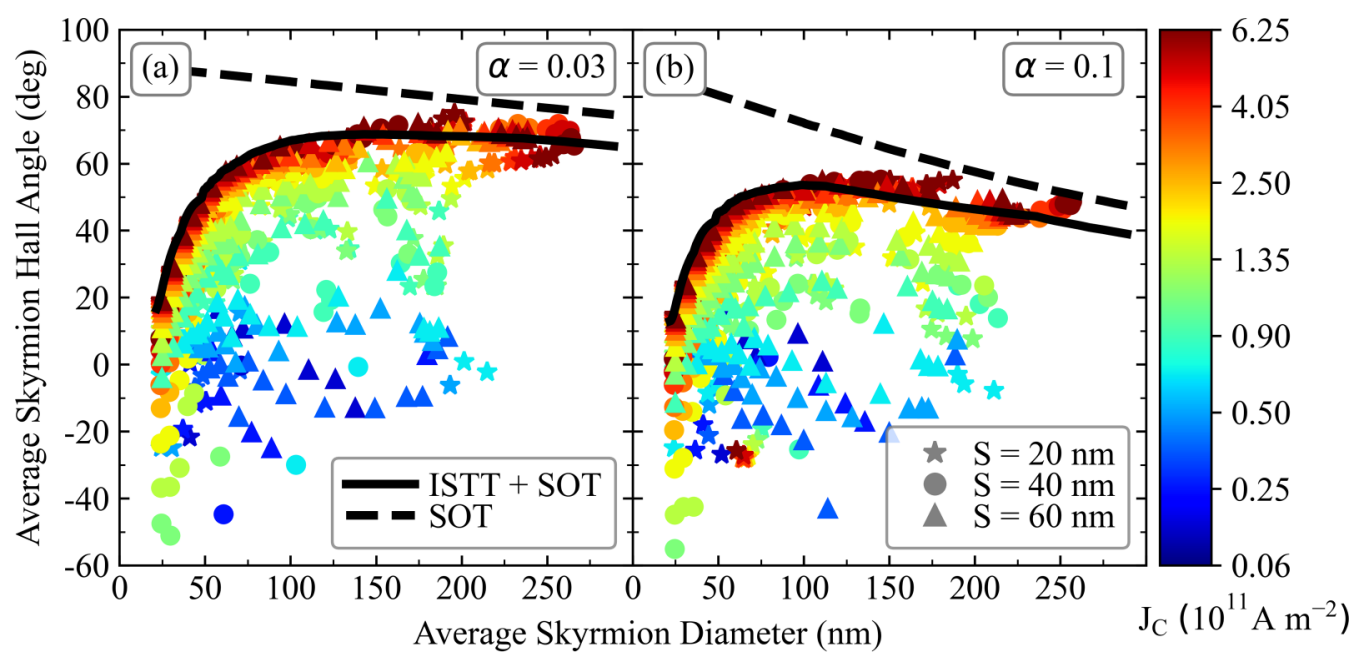

FIG. 3. Skyrmion Hall angle dependence on skyrmion diameter for damping values of (a) $\alpha=0.03$ and (b) $\alpha=0.1$, with varying surface disorder periodicity in a Co $(1 \mathrm{~nm}) / \mathrm{Pt}(3 \mathrm{~nm})$ bilayer. Star, circle, and triangle correspond to 20-, 40-, and 60-nm disorder periods, respectively. The color map corresponds to the applied current density in the $+x$ direction of the Co/Pt system. The solid black lines display the full spin transport solver results in the ideal case. The dashed black lines show the SOT dependence obtained from the Thiele equation solution with $u_{\mathrm{STT}}=0$.

spacing, respectively, between peaks and troughs. Figure 1(b) shows a disorder variation period of $60 \mathrm{~nm}$. Furthermore, in the ideal case a single applied current was used as the SkHA values are independent of the applied current density, but in the disordered case a range of applied current densities were used, ranging from $J_{c}=6 \mathrm{GA} \mathrm{m}^{-2}$ up to $J_{c}=625 \mathrm{GA} \mathrm{m}^{-2}$.

Figure 1(b) shows three simulated skyrmion paths which display three regimes: pinned, creep, and flow regime. These have been defined when being driven by current as (i) the pinned regime in which the skyrmion is confined within a potential well due to the energy landscape surrounding the skyrmion, and displays an orbiting path inside the pinning potential; (ii) the creep regime, in which the skyrmion has a large enough driving force to overcome the energy barrier and unpin itself; however, the skyrmion is heavily affected by the local energy landscape resulting in a significantly distorted path via sporadic jumps between pinning sites; (iii) the flow regime, in which the skyrmion displays a near-linear path across the disorder landscape, close to that obtained in the ideal case.

Figure 3 shows the relationship between the SkHA and skyrmion diameter for $\alpha=0.03$ and $\alpha=0.1$. The solid black line is the trend obtained in the ideal case for the full spin torque, and the dashed black line shows the expected behavior under SOT using the rigid skyrmion model. The SkHA increases rapidly with skyrmion diameter up to $100 \mathrm{~nm}$, after which a flattening of the trend occurs. The maximum SkHA obtained is $\theta_{\mathrm{SkHA}}=75^{\circ}$ for $\alpha=0.03$, and $\theta_{\mathrm{SkHA}}=55^{\circ}$ for $\alpha=0.1$. At small driving currents skyrmions experience high deflection due to the local energy landscape, which is typical for the creep regime [61] where skyrmion motion occurs via sporadic jumps between pinning sites. In this regime, the SkHA is nearly independent of the skyrmion diameter, in agreement with experimental observations [14]. At larger driving currents a more defined SkHA behavior becomes apparent, tending towards the ideal case and entering the flow regime. There are two distinct regions at larger driving cur- rents: (i) skyrmions with diameters of $100 \mathrm{~nm}$ and less which show a rapid SkHA increase with increasing diameters due to the combined action of the SOT and ISTT, and (ii) skyrmions with diameters greater than $100 \mathrm{~nm}$ that show a saturation of the SkHA, which becomes near constant. Thus the effect of the combined SOT and ISTT model is in sharp contrast to the Thiele equation solution in Eq. (9) with $u_{\text {STT }}=0$, which predicts a monotonic increase in the SkHA with decreasing skyrmion diameter.

Reference [14] observed skyrmion characteristics experimentally, analyzed using a range of damping factors. Using the Thiele equation with SOT only, significant discrepancies are observed between modeling and experimental results. In particular, the experimental results show a SkHA largely independent of skyrmion diameter, whereas the model based on the Thiele equation predicts a significant increase in SkHA with decreasing skyrmion diameter, and this discrepancy is significantly pronounced below a diameter of $100 \mathrm{~nm}$ and has not been reproduced using micromagnetics simulations to date. The results obtained here show the ISTT contribution is particularly important for small skyrmion diameters, which can lead to a near diameter-independent SkHA as observed experimentally, particularly when defect energy landscapes are taken into consideration.

Reference [16] shows interesting skyrmion temperature characteristics in which skyrmion velocity is strongly dependent on temperature due to the increase in the temperaturedependent DL SOT. However, the temperature is shown to have little effect on the SkHA, based on a model of spin structure deformations due to the FL SOT, as the relationship between velocity and SkHA collapse onto a universal trend line. This behavior has also been simulated [28] in which the skyrmion path and SkHA deviate insignificantly at $T=0 \mathrm{~K}$ and $T=297 \mathrm{~K}$ in a $\mathrm{Pt} / \mathrm{Co} / \mathrm{Ta}$ stack. When considering both ISTT and SOT components in our model the SkHA and skyrmion velocity show similar characteristics to the experimental results, as shown in Fig. 4. This shows the SkHA 


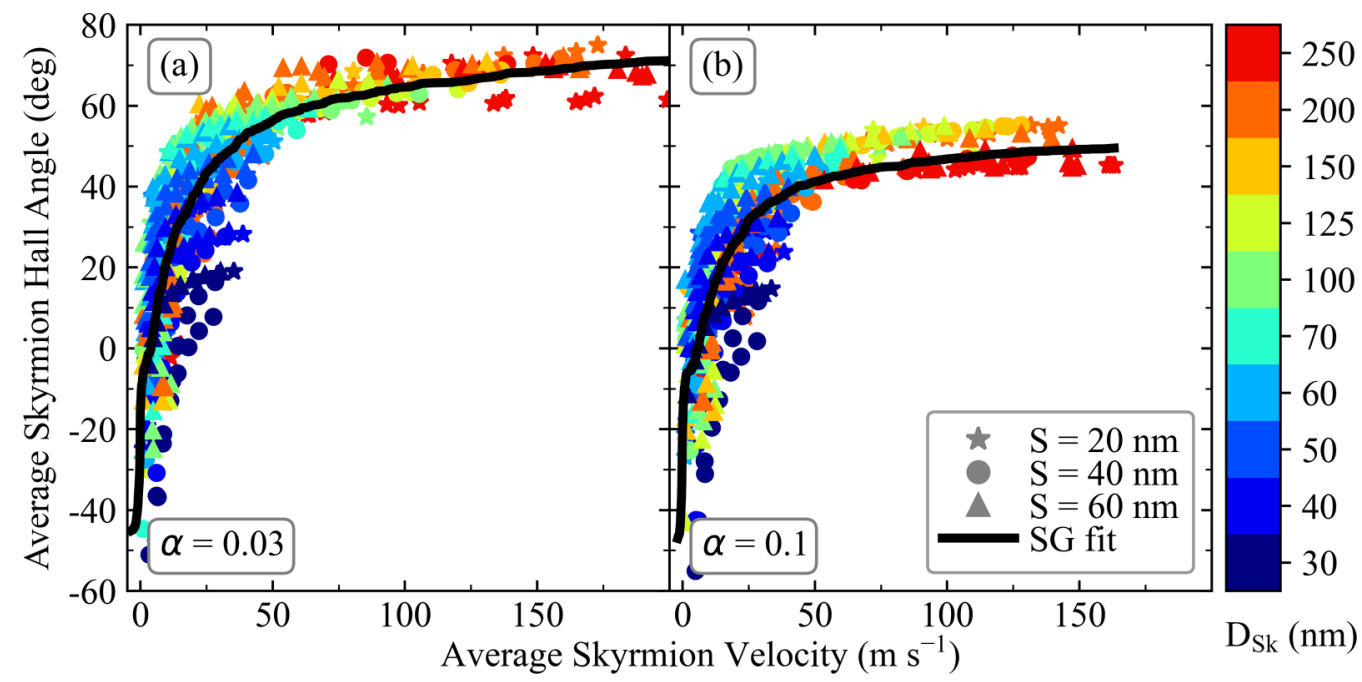

FIG. 4. Skyrmion Hall angle dependence on the skyrmion velocity for three disorder variation periods. Two damping values in (a) $\alpha=0.03$ and (b) $\alpha=0.1$ were used to obtain the dependence. The color map details the diameter bin ranges from the minimum range of less than $30 \mathrm{~nm}$ up to a maximum of $250 \mathrm{~nm}$. The points labeled in (a), (b) correspond to the disorder variation periods on the $\operatorname{Co}(1 \mathrm{~nm}) / \mathrm{Pt}(3 \mathrm{~nm}) \mathrm{bilayer}$. Star, circle, and triangle correspond to 20-, 40-, and 60-nm spacings, respectively. The solid black line shows the Savitzky-Golay trend line.

as a function of the skyrmion velocity, with the skyrmion diameter color coded as shown in the legend, for the three different disorder variation periods. The black line shows the Savitzky-Golay fit to the data to illustrate the average trend of the SkHA. There are two distinct velocity behaviors apparent in Fig. 4. Initially, there is a steep increase in the SkHA with an increasing velocity at mainly small skyrmion diameters, indicative of the creep regime, which agrees well with established defect theory [18] and experimental works [14,16,19]. Following this initial steep increase in SkHA, a flattening of the behavior occurs in which a much more gradual increase is observed over a much larger velocity range. These results for a range of skyrmion diameters suggest a collapse onto a universal SkHA versus skyrmion velocity dependence which is nearly independent of the surface roughness characteristics, similar to experimental results obtained in similar systems $[14,16,18,19]$.

Figure 5 shows the velocity dependence on the applied current. The relationship for the ideal case is shown by the color-coded dashed lines. A current-velocity relationship is subsequently established for both $\alpha=0.03$ and 0.1 , indicating a monotonic increase of the average skyrmion velocity, dependent on the applied current density. The disordered simulations correspond very well to simulations of no disorder in which the effect of surface roughness mainly affects the SkHA by deflecting skyrmions and does not have an appreciable influence on the skyrmion velocity. Furthermore, the damping values have a significant effect on the skyrmion

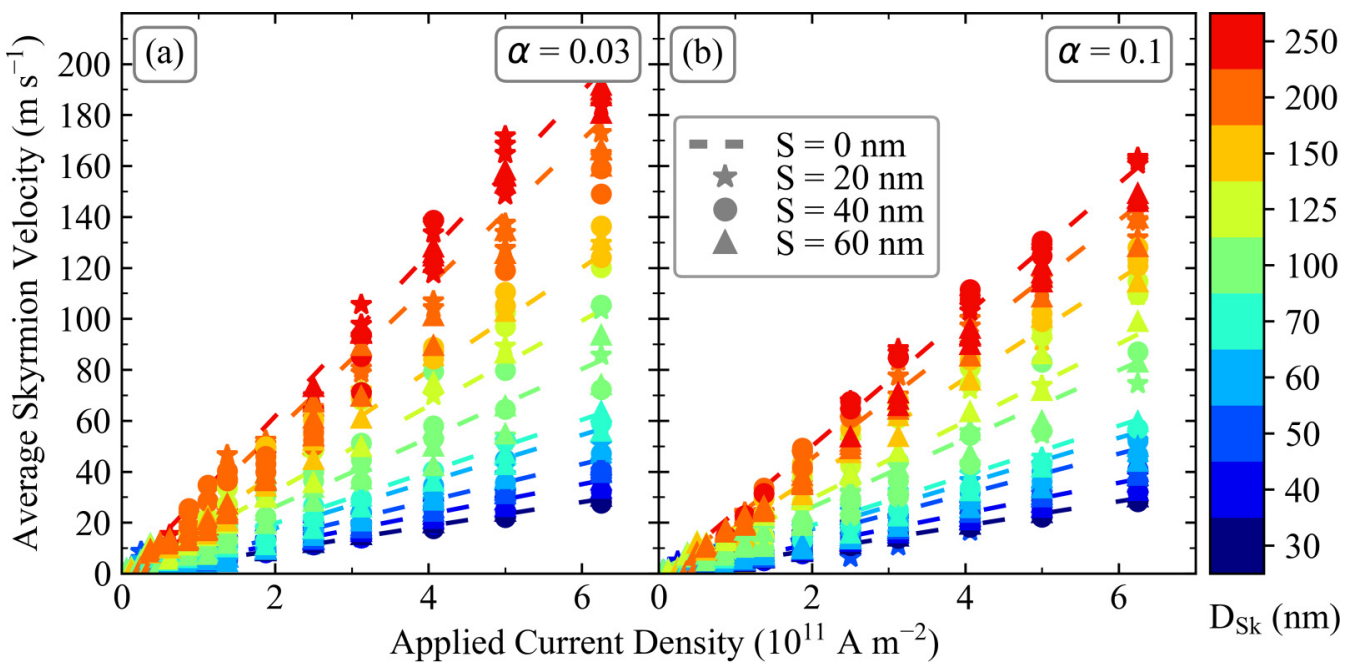

FIG. 5. Relationship between skyrmion velocity and applied current density for damping values of (a) $\alpha=0.03$ and (b) $\alpha=0.1$, with surface disorder periodicity in a Co $(1 \mathrm{~nm}) / \mathrm{Pt}(3 \mathrm{~nm})$ bilayer. Star, circle, and triangle correspond to 20-, 40-, and 60-nm disorder periods, respectively. Each skyrmion was assigned into a bin with assigned diameter ranges. The color key details the diameter bin ranges from $30 \mathrm{~nm}$ up to a maximum of $250 \mathrm{~nm}$. The dashed lines correspond to the relationship for the ideal $(S=0 \mathrm{~nm})$ case at each diameter range. 


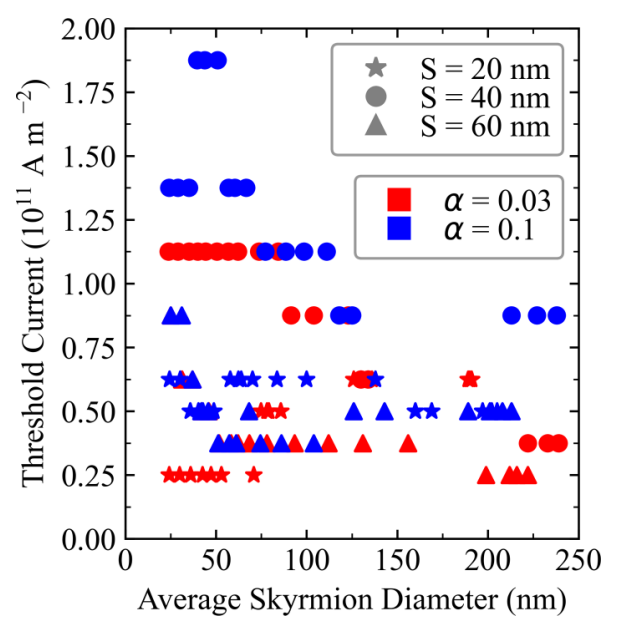

FIG. 6. Skyrmion threshold current for damping values $\alpha=0.03$ and $\alpha=0.1$ represented by the solid and dashed lines, respectively. Varying applied disorder variation periods of 20, 40, and $60 \mathrm{~nm}$ were also used which are represented in red, green, and blue, respectively.

velocity which is predicted by the Thiele equation and is most evident in the 250-nm bin at the largest applied current, where for $\alpha=0.03$ and $\alpha=0.1$ the skyrmion velocities are $v_{\mathrm{Sk}}=191 \mathrm{~m} \mathrm{~s}^{-1}$ and $v_{\mathrm{Sk}}=163 \mathrm{~m} \mathrm{~s}^{-1}$, respectively. As pinned skyrmions are not shown, the skyrmion velocity at low driving current displays slight stochasticity for $J_{c}<1 \times 10^{11} \mathrm{~A} \mathrm{~m}^{-2}$, after which the skyrmions have a well-defined velocity with respect to the applied current. Furthermore, for $J_{c}>2.5 \times$ $10^{11} \mathrm{~A} \mathrm{~m}^{-2}$ the skyrmion velocity tends to the linear profile of the disorder-free case. This behavior corresponds well with experimental behavior [18,19,59]. A more detailed depiction of the velocity-current-density relationship at small current densities is shown in Appendix C.

Figure 6 shows the relationship between the skyrmion threshold current and skyrmion diameter for $\alpha=0.03$ and $\alpha=0.1$. We define the threshold current as the current required to change the skyrmion from the pinned regime to the creep regime which has been referred to as the depinning current. For $\alpha=0.1$ the required current to unpin the skyrmions is larger than for $\alpha=0.03$ at small skyrmion diameters; however, for larger diameters the threshold currents for both $\alpha=0.1$ and $\alpha=0.03$ tend towards each other. Typically for small skyrmions, the threshold current density is significantly greater than for larger skyrmions, which is most likely due to greater skyrmion rigidity at smaller diameters. Thus, as the diameter increases the skyrmions become more malleable and start to deform as diametrically opposite points on the skyrmion border experience different pinning energies. This helps to reduce the threshold current, as the exchange energy built up through skyrmion deformations assists in depinning the skyrmion border for skyrmions much larger than the disorder variation period.

\section{CONCLUSIONS}

We have studied single skyrmion motion in ultrathin $\mathrm{Pt} / \mathrm{Co}$ bilayers with and without disorder for a large range of skyrmion diameters utilizing micromagnetics simulations coupled with a self-consistent spin transport solver. As well
TABLE I. Material parameters used to model the Pt/Co bilayer.

\begin{tabular}{lcc}
\hline \hline Parameter & Value & References \\
\hline$|\mathrm{D}|(\mathrm{Co})$ & $1.5 \mathrm{~mJ} \mathrm{~m}^{-2}$ & {$[12]$} \\
$M_{S}(\mathrm{Co})$ & $600 \mathrm{kA} \mathrm{m}^{-1}$ & {$[12]$} \\
$A(\mathrm{Co})$ & $10 \mathrm{pJ} \mathrm{m}^{-1}$ & {$[12]$} \\
$K_{u}(\mathrm{Co})$ & $380 \mathrm{~kJ} \mathrm{~m}^{-3}$ & {$[12]$} \\
$\alpha(\mathrm{Co})$ & $0.03,0.1$ & {$[46-52]$} \\
$\mathrm{g}_{\text {rel }}(\mathrm{Co})$ & 1.3 & {$[62]$} \\
$\sigma(\mathrm{Co})$ & $5 \mathrm{MS} \mathrm{m}^{-1}$ & {$[63]$} \\
$\mathrm{De}(\mathrm{Co})$ & $0.0012 \mathrm{~m}^{2} \mathrm{~s}^{-1}$ & {$[63]$} \\
$\mathrm{De}(\mathrm{Pt})$ & $0.004 \mathrm{~m}^{2} \mathrm{~s}^{-1}$ & {$[63]$} \\
$\lambda_{\text {sf }}(\mathrm{Co})$ & $42 \mathrm{~nm}$ & {$[63,64]$} \\
$\lambda_{\mathrm{J}}(\mathrm{Co})$ & $2 \mathrm{~nm}(\mathrm{Co})$ & {$[65]$} \\
$\lambda_{\phi}(\mathrm{Co})$ & $3.2 \mathrm{~nm}$ & {$[66]$} \\
$\lambda_{\text {sf }}(\mathrm{Pt})$ & $1.4 \mathrm{~nm}^{-1}$ & {$[30]$} \\
$\sigma(\mathrm{Pt})$ & $7 \mathrm{MS} \mathrm{m}^{-1}$ & {$[30]$} \\
$\operatorname{Re}\left(\mathrm{G}^{\uparrow \downarrow}\right)(\mathrm{Pt} / \mathrm{Co})$ & $1.5 \mathrm{PS} \mathrm{m}^{-2}$ & {$[30]$} \\
$\operatorname{Im}\left(\mathrm{G}^{\uparrow} \downarrow\right)(\mathrm{Pt} / \mathrm{Co})$ & $0.45 \mathrm{PS} \mathrm{m}^{-2}$ & {$[30]$} \\
$\theta_{\mathrm{SHA}}(\mathrm{Pt})$ & 0.19 & $\mathrm{a}$ \\
\hline \hline
\end{tabular}

${ }^{\text {a Value is obtained using Ref. [19] in conjunction with Eq. (6) which }}$ can be solved for $\operatorname{Im}\left(G^{\uparrow \downarrow}\right)$.

as SOT acting on the Co layer arising from the SHE, the ISTT was also shown to have a significant contribution to skyrmion motion. The included ISTT term was shown to significantly reduce the discrepancy for the SkHA between experimental results in the literature and SOT-only modeling for skyrmion diameters less than $100 \mathrm{~nm}$. The SOT-only model is shown to be in agreement for large skyrmions, while for smaller skyrmions the inclusion of the additional ISTT term results in decreasing SkHA with decreasing skyrmion diameter. The analysis conducted in this work can also be extended to a collection of skyrmions in a sample which will be of interest in future work. The results obtained for a range of skyrmion diameters are shown to collapse onto a universal SkHA-velocity dependence, nearly independent of the surface roughness characteristics. Our results shed light on the current-induced skyrmion dynamics for a large range of diameters and highlight the current shortfalls of using a purely SOT based model in micromagnetics modeling.

\section{METHODS}

All simulations were done using BORIS COMPUTATIONAL SPINTRONICS software [55], version 2.6. Material parameters used in the simulations are summarized in Table I.

Computations were done using cell-centered finite difference discretization. For magnetization dynamics, the computational cell size used was $(2,2,1 \mathrm{~nm})$. For spin transport calculations the computation cell size was $(2,2,0.5 \mathrm{~nm})$ for both the Co and Pt layers. The LLG equation was evaluated using the RKF45 evaluation which is an adaptive time step method. Initial relaxation was evaluated using the steepest descent method. All computations were completed on the GPU using the CUDA C framework. Roughness profiles were generated using a jagged granular generator algorithm. Equally spaced coefficients at 20,40, and $60 \mathrm{~nm}$ spacings in the $x y$ plane are randomly generated. The remaining coefficients 


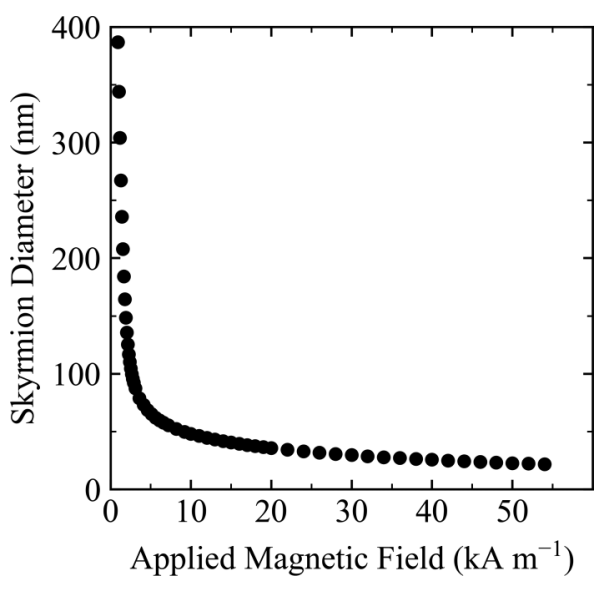

FIG. 7. Skyrmion diameter dependence on out-of-plane magnetic field, computed for the ideal case.

are obtained using bilinear interpolation from the randomly generated points.

The Dzyaloshinskii-Moriya interfacial (iDMI) exchange interaction contribution in the $x y$ plane is included as an effective field contribution, which adds to the direct exchange contribution, and is given by

$$
\mathbf{H}=-\frac{2 D}{\mu_{0} M_{S}^{2}}\left(\frac{\partial M_{z}}{\partial x}, \frac{\partial M_{z}}{\partial y},-\frac{\partial M_{x}}{\partial x}-\frac{\partial M_{y}}{\partial y}\right) .
$$

\section{APPENDIX A: SKYRMION DIAMETER DEPENDENCE ON THE APPLIED MAGNETIC FIELD}

To obtain Fig. 7 an isolated skyrmion was set in the center of a disorder-free Co $(1 \mathrm{~nm}) / \mathrm{Pt}(3 \mathrm{~nm})$ bilayer and allowed to relax. Relaxation of the skyrmion is accomplished by evaluating the LLG with the steepest descent method. After the relaxation stage, under a small applied field, was satisfied, an out-of-plane $(+z$ direction) magnetic field was applied and the skyrmion was allowed to relax under the new field. After this, the skyrmion diameter was obtained in the $x$ and $y$ direction independently and averaged.

\section{APPENDIX B: DEPENDENCE OF FITTING PARAMETERS ON SKYRMION DIAMETERS}

Figures 8(a) and 8(b) show the effective spin Hall angle and fieldlike SOT fitting parameters for a range of skyrmion diameters, respectively. Figures 8(c) and 8(d) show the dependence of the effective spin polarization and nonadiabaticity parameters on skyrmion diameter, respectively. There is a slight deviation at small diameters away from the constant value at large diameters. Where dependence is evident, smaller $G_{r}$ ratios provide a larger effect.

To obtain Fig. 8 a disorder-free $\operatorname{Co}(1 \mathrm{~nm}) / \mathrm{Pt}(3 \mathrm{~nm})$ bilayer was set using the material parameters stated in Table I. An isolated skyrmion was then created in the center of the Co surface, controlled by an out-of-plane magnetic field to tune the skyrmion diameter, and allowed to relax using the steepest descent method. The values for the fitting parameters could then be obtained. Initially, the spin accumulation was computed using the spin transport solver which is used to obtain

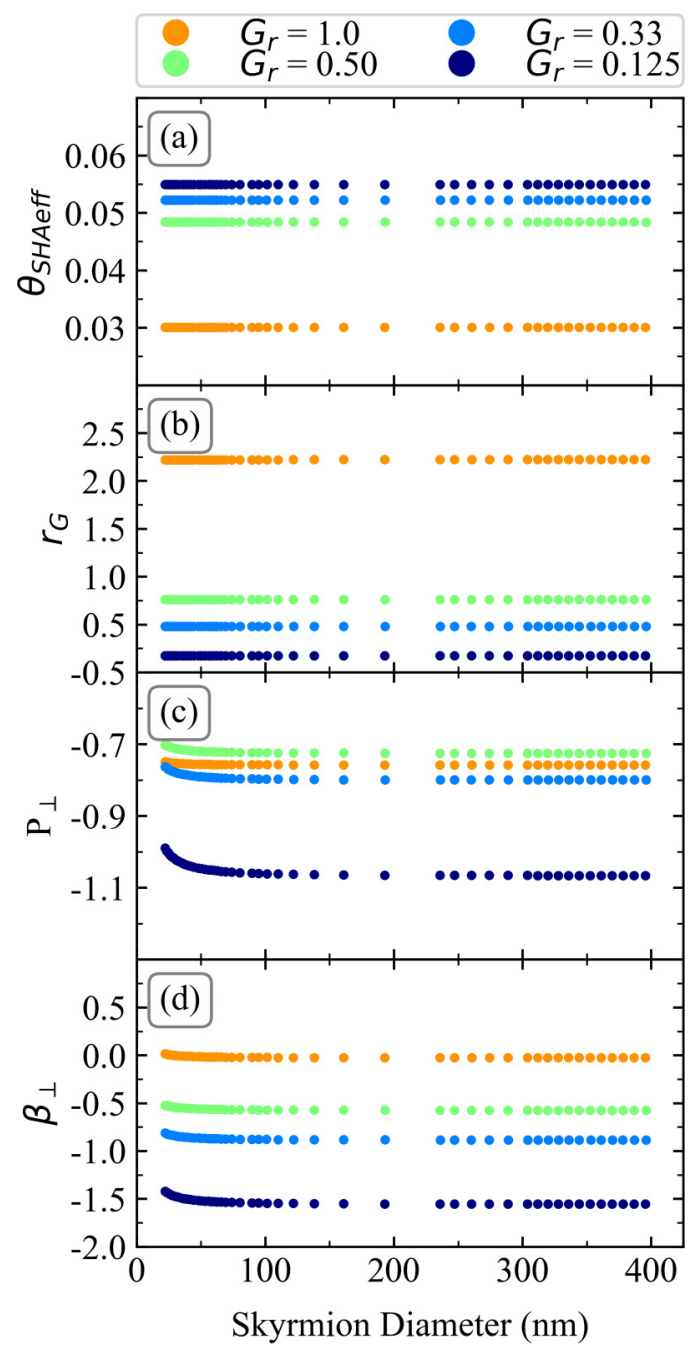

FIG. 8. The dependence of modeling (a) effective Spin Hall angle, (b) fieldlike spin coefficient, (c) effective spin polarization, and (d) effective nonadiabaticity parameters, respectively, on skyrmion diameter in a disorder-free $\mathrm{Co} / \mathrm{Pt}$ bilayer for a range of imaginary to real spin mixing conductance ratios, $G_{r}$.

the total spin torque. To obtain $\theta_{\text {SHAeff }}$ and $\mathrm{r}_{G}$, Eq. (4) is fitted to the total spin torque when negating the ISTT contribution ( $P$ set to zero). Similarly, using Eq. (7), $P_{\perp}$ and $\beta_{\perp}$ can be obtained again as fitting parameters when negating the SOT contribution ( $\theta_{\mathrm{SHA}}$ set to zero). As we have verified, the full spin torque computed self-consistently is reproduced using the SOT and ISTT torques with the four fitting parameters to $R$ values better than 0.99 .

\section{APPENDIX C: VELOCITY-CURRENT-DENSITY RELATIONSHIP AT SMALL CURRENT DENSITIES}

Figure 9 shows the skyrmion velocity for small driving currents which is not visible in Fig. 5 due to the large range. The skyrmion velocity for this range shows stochastic behavior, and as a result, does not correspond well with the ideal simulations displayed by the colored dashed lines. 


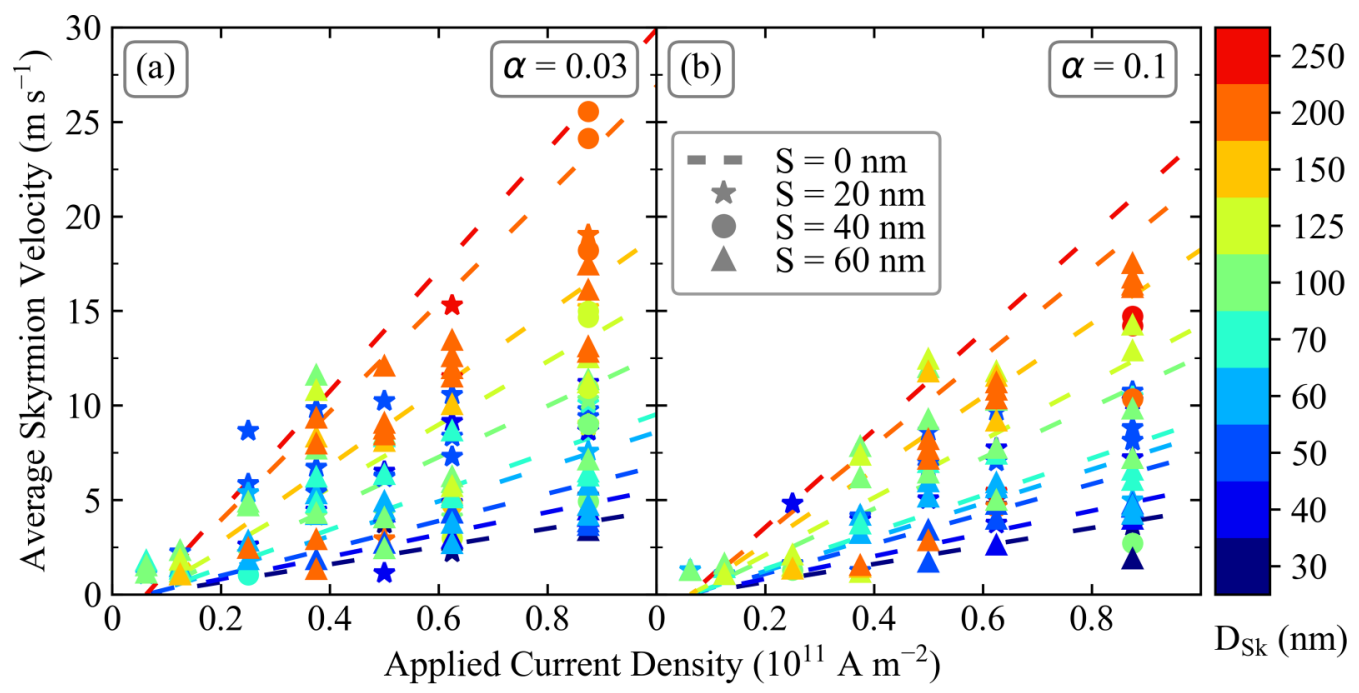

FIG. 9. The velocity dependence on applied current density for (a) $\alpha=0.03$ and (b) $\alpha=0.01$. This is a truncated version of Fig. 5 so the stochastic behavior at lower applied current densities can be seen. The color key details the skyrmion diameter bin ranges for the minimum of less than $30 \mathrm{~nm}$ up to a maximum of $250 \mathrm{~nm}$. The dashed lines in (a,b) correspond to the relationship for the ideal case $(S=0 \mathrm{~nm})$ at each diameter range.

[1] A. N. Bogdanov and D. A. Yablonskii, Thermodynamically stable "vortices" in magnetically ordered crystals. The mixed state of magnets, Zh. Eksp. Teor. Fiz. 95, 178 (1989) [Sov. Phys. JETP 68, 101 (1989)].

[2] K. Everschor-Sitte, J. Masell, R. M. Reeve, and M. Kläui, Perspective: Magnetic skyrmions-Overview of recent progress in an active research field, J. Appl. Phys. 124, 240901 (2018).

[3] A. Fert, N. Reyren, and V. Cros, Magnetic skyrmions: Advances in physics and potential applications, Nat. Rev. Mater. 2, 17031 (2017).

[4] R. Ramaswamy, J. M. Lee, K. Cai, and H. Yang, Recent advances in spin-orbit torques: Moving towards device applications, Appl. Phys. Rev. 5, 031107 (2018).

[5] A. Soumyanarayanan, M. Raju, A. L. Gonzalez Oyarce, A. K. C. Tan, M.-Y. Im, A. P. Petrović, P. Ho, K. H. Khoo, M. Tran, C. $\mathrm{K}$. Gan et al., Tunable room-temperature magnetic skyrmions in $\mathrm{Ir} / \mathrm{Fe} / \mathrm{Co} / \mathrm{Pt}$ multilayers, Nat. Mater. 16, 898 (2017).

[6] D. Maccariell, W. Legrand, N. Reyren, K. Garcia, K. Bouzehouane, S. Collin, V. Cros, and A. Fert, Electrical detection of single magnetic skyrmions in metallic multilayers at room temperature, Nat. Nanotechnol. 13, 233 (2018).

[7] X. Z. Yu, N. Kanazawa, Y. Onose, K. Kimoto, W. Z. Zhang, S. Ishiwata, Y. Matsui, and Y. Tokura, Near room-temperature formation of a skyrmion crystal in thin-films of the helimagnet FeGe, Nat. Mater. 10, 106 (2011).

[8] G. Yu, A. Jenkins, X. Ma, S. A. Razavi, C. He, G. Yin, Q. Shao, Q. L. He, H. Wu, W. Li et al., Room-temperature skyrmions in an antiferromagnet-based heterostructure, Nano Lett. 18, 980 (2018).

[9] C. Moreau-Luchaire, C. Moutafis, N. Reyren, J. Sampaio, C. A. F. Vaz, N. Van Horne, K. Bouzehouane, K. Garcia, C. Deranlot, P. Warnicke et al., Additive interfacial chiral interaction in multilayers for stabilization of small individual skyrmions at room temperature, Nat. Nanotechnol. 11, 444 (2016).
[10] I. Dzyaloshinsky, A thermodynamic theory of weak ferromagnetism of antiferromagnetics, J. Phys. Chem. Solids 4, 241 (1958).

[11] T. Moriya, Anisotropic superexchange interaction and weak ferromagnetism, Phys. Rev. 120, 91 (1960).

[12] S. Woo, K. Litzius, B. Krüger, M. Im, L. Caretta, K. Richter, M. Mann, A. Krone, R. M. Reeve, M. Weigand et al., Observation of room-temperature magnetic skyrmions and their current-driven dynamics in ultrathin metallic ferromagnets, Nat. Mater. 15, 501 (2016).

[13] S. Woo, K. M. Song, X. Zhang, Y. Zhou, M. Ezawa, X. Liu, S. Finizio, J. Raabe, N. J. Lee, S. Kim et al., Current-driven dynamics and inhibition of the skyrmion Hall effect of ferrimagnetic skyrmions in GdFeCo films, Nat. Commun. 9, 959 (2018).

[14] K. Zeissler, S. Finizio, C. Barton, A. J. Huxtable, J. Massey, J. Raabe, A. V. Sadovnikov, S. A. Nikitov, R. Brearton, T. Hesjedal et al., Diameter-independent skyrmion Hall angle observed in chiral magnetic multilayers, Nat. Commun. 11, 428 (2020).

[15] K. Litzius, I. Lemesh, B. Krüger, P. Bassirian, L. Carette, K. Richter, F. Büttner, K. Sato, O. A. Tretiakov, K. Förster et al., Skyrmion Hall effect revealed by direct time-resolved x-ray microscopy, Nat. Phys. 13, 170 (2017).

[16] K. Litzius, J. Leliaert, P. Bassirian, D. Rodrigues, S. Kromin, I. Lemesh, J. Zazvorka, K. Lee, J. Mulkers, N. Kerber et al., The role of temperature and drive current in skyrmion dynamics, Nat. Electron. 3, 30 (2020).

[17] W. Legrand, D. Maccariello, N. Reyren, K. Garcia, C. Moutafis, C. Moreau-Luchaire, S. Collin, K. Bouzehouane, V. Cros, and A. Fert, Room-temperature current-induced generation and motion of sub-100 nm skyrmions, Nano Lett. 17, 2703 (2017).

[18] W. Jiang, X. Zhang, G. Yu, W. Zhang, X. Wang, M. B. Jungfleisch, J. E. Pearson, X. Cheng, O. Heinonen, K. L. 
Wang et al., Direct observation of the skyrmion Hall effect, Nat. Phys. 13, 162 (2017).

[19] R. Juge, S. Je, D. Chaves, L. D. Buda-Prejbeanu, J. Peña-Garcia, J. Nath, I. M. Miron, K. G. Rana, L. Aballe, M. Foerster et al., Current-Driven Skyrmion Dynamics and DriveDependent Skyrmion Hall Effect in an Ultrathin Film, Phys. Rev. Appl. 12, 044007 (2019).

[20] P. P. J. Haazen, E. Murè, J. H. Franken, R. Lavrijsen, H. J. M. Swagten, and B. Koopmans, Domain wall depinning governed by the spin Hall effect, Nat. Mater. 12, 299 (2013).

[21] S. Emori, U. Bauer, S. M. Ahn, E. Martinez, and G. S. D. Beach, Current-driven dynamics of chiral ferromagnetic domain walls, Nat. Mater. 12, 611 (2013).

[22] C. F. Pail, L. Liu, Y. Li1, H. W. Tseng, D. C. Ralph, and R. A. Buhrman, Spin transfer torque devices utilizing the giant spin Hall effect of tungsten, Appl. Phys. Lett. 101, 122404 (2012).

[23] L. Liu, C. F. Pai, Y. Li, H. W. Tseng, D. C. Ralph, and R. A. Buhrman, Spin-torque switching with the giant spin Hall effect of tantalum, Science 336, 555 (2012).

[24] L. Liu, O. J. Lee, T. J. Gudmundsen, D. C. Ralph, and R. A. Buhrman, Current-Induced Switching of Perpendicularly Magnetized Magnetic Layers Using Spin Torque from the Spin Hall Effect, Phys. Rev. Lett. 109, 096602 (2012).

[25] C. Reichhardt, D. Ray, and C. J. Olson Reichhardt, Collective Transport Properties of Driven Skyrmions with Random Disorder, Phys. Rev. Lett. 114, 217202 (2015).

[26] J. Plettenberg, M. Stier, and M. Thorwart, Steering of the Skyrmion Hall Angle by Gate Voltages, Phys. Rev. Lett. 124, 207202 (2020).

[27] C. A. Akosa, H. Li, G. Tatara, and O. A. Tretiakov, Tuning the Skyrmion Hall Effect via Engineering of Spin-Orbit Interaction, Phys. Rev. Appl. 12, 054032 (2019).

[28] S. Lepadatu, Effect of inter-layer spin diffusion on skyrmion motion in magnetic multilayers, Sci. Rep. 9, 9592 (2019).

[29] A. Brataas, Y. U. V. Nazarov, and G. E. W. Bauer, FiniteElement Theory of Transport in Ferromagnet-Normal Metal Systems, Phys. Rev. Lett. 84, 2481 (2000).

[30] W. Zhang, W. Han, X. Jiang, S. H. Yang, and S. S. P. Parkin, Role of transparency of platinum-ferromagnet interfaces in determining the intrinsic magnitude of the spin Hall effect, Nat. Phys. 11, 496 (2015).

[31] S. Zhang and Z. Li, Roles of Nonequilibrium Conduction Electrons on the Magnetization Dynamics of Ferromagnets, Phys. Rev. Lett. 93, 127204 (2004).

[32] Z. Li and S. Zhang, Domain-wall dynamics driven by adiabatic spin-transfer torques, Phys. Rev. B 70, 024417 (2004).

[33] S.-G. Je, S.-C. Yoo, J.-S. Kim, Y.-K. Park, M.-H. Park, J. Moon, B.-C. Min, and S.-B. Choe, Emergence of Huge Negative SpinTransfer Torque in Atomically Thin Co layers, Phys. Rev. Lett. 118, 167205 (2017).

[34] A. A. Thiele, Steady-State Motion of Magnetic Domains, Phys. Rev. Lett. 30, 230 (1973).

[35] K. Everschor, M. Garst, R. A. Duine, and A. Rosch, Currentinduced rotational torques in the skyrmion lattice phase of chiral magnets, Phys. Rev. B 84, 064401 (2011).

[36] B. S. Kim, Modeling Hall viscosity in magnetic-skyrmion systems, Phys. Rev. Res. 2, 013268 (2020).

[37] W. Jiang, G. Chen, K. Liu, J. Zang, S. G. E. te Velthuis, and A. Hoffmann, Skyrmions in magnetic multilayers, Phys. Rep. 704, 1 (2017)
[38] A. Hrabec, J. Sampaio, M. Belmeguenai, I. Gross, R. Weil, S. M. Chérif, A. Stashkevich, V. Jacques, A. Thiaville, and S. Rohart, Current-induced skyrmion generation and dynamics in symmetric bilayers, Nat. Commun. 8, 15765 (2017).

[39] H. Yang, A. Thiaville, S. Rohart, A. Fert, and M. Chshiev, Anatomy of Dzyaloshinskii-Moriya Interaction at $\mathrm{Co} / \mathrm{Pt}$ Interfaces, Phys. Rev. Lett. 115, 267210 (2015).

[40] H. Yang, O. Boulle, V. Cros, A. Fert, and M. Chshiev, Controlling Dzyaloshinskii-Moriya interaction via chirality dependent atomic-layer stacking, insulator capping and electric field, Sci. Rep. 8, 12356 (2018).

[41] D. Claudio-Gonzalez, A. Thiaville, and J. Miltat, Domain Wall Dynamics under Nonlocal Spin-Transfer Torque, Phys. Rev. Lett. 108, 227208 (2012).

[42] A. Bisig, C. A. Akosa, J. H. Moon, J. Rhensius, C. Moutafis, A. von Bieren, J. Heidler, G. Kiliani, M. Kammerer, M. Curcic et al., Enhanced Nonadiabaticity in Vortex Cores due to the Emergent Hall Effect, Phys. Rev. Lett. 117, 277203 (2016).

[43] J. Dubowik, P. Graczyk, A. Krysztofik, H. Głowiński, E. Coy, K. Załeski, and I. Gościańska, Non-Negligible Imaginary Part of the Spin-Mixing Conductance and its Impact on Magnetization Dynamics in Heavy-Metal-Ferromagnet Bilayers, Phys. Rev. Appl. 13, 054011 (2020)

[44] K.-W. Kim, Spin transparency for the interface of an ultrathin magnet within the spin dephasing length, Phys. Rev. B 99, 224415 (2019).

[45] S. J. Yuan, L. Sun, H. Sang, J. Du, and S. M. Zhou, Interfacial effects on magnetic relaxation in $\mathrm{Co} / \mathrm{Pt}$ multilayers, Phys. Rev. B 68, 134443 (2003).

[46] I. Benguettat-El Mokhtari, A. Mourkas, P. Ntetsika, I. Panagiotopoulos, Y. Roussigné, S. M. Cherif, A. Stashkevich, F. Kail, L. Chahed, and M. Belmeguenai, Interfacial Dzyaloshinskii-Moriya interaction, interface-induced damping and perpendicular magnetic anisotropy in $\mathrm{Pt} / \mathrm{Co} / \mathrm{W}$ based multilayers, J. Appl. Phys. 126, 133902 (2019).

[47] J. M. L. Beaujour, W. Chen, K. Krycka, C. C. Kao, J. Z. Sun, and A. D. Kent, Ferromagnetic resonance study of sputtered Co|Ni multilayers, Eur. Phys. J. B 59, 475 (2007).

[48] E. Barati, M. Cinal, D. M. Edwards, and A. Umerski, Gilbert damping in magnetic layered systems, Phys. Rev. B 90, 014420 (2014).

[49] A. J. Schellekens, L. Deen, D. Wang, J. T. Kohlhepp, H. J. M. Swagten, and B. Koopmans, Determining the Gilbert damping in perpendicularly magnetized $\mathrm{Pt} / \mathrm{Co} / \mathrm{AlO}_{x}$ films, Appl. Phys. Lett. 102, 082405 (2013).

[50] A. Barman, S. Wang, O. Hellwig, A. Berger, E. E. Fullerton, and $\mathrm{H}$. Schmidt, Ultrafast magnetization dynamics in high perpendicular anisotropy $[\mathrm{CoPt}]_{n}$ multilayers, J. Appl. Phys. 101, 09D102 (2007).

[51] S. Mizukami, E. P. Sajitha, D. Watanabe, F. Wu, T. Miyazaki, H. Naganuma, M. Oogane, and Y. Ando, Gilbert damping in perpendicularly magnetized $\mathrm{Pt} / \mathrm{Co} / \mathrm{Pt}$ films investigated by alloptical pump-probe technique, Appl. Phys. Lett. 96, 152502 (2010).

[52] P. J. Metaxas, J. P. Jamet, A. Mougin, M. Cormier, J. Ferré, V. Baltz, B. Rodmacq, B. Dieny, and R. L. Stamps, Creep and Flow Regimes of Magnetic Domain-Wall Motion in Ultrathin $\mathrm{Pt} / \mathrm{Co} / \mathrm{Pt}$ Films with Perpendicular Anisotropy, Phys. Rev. Lett. 99, 217208 (2007). 
[53] C. A. Akosa, P. B. Ndiaye, and A. Manchon, Intrinsic nonadiabatic topological torque in magnetic skyrmions and vortices, Phys. Rev. B 95, 054434 (2017).

[54] C. A. Akosa, O. A. Tretiakov, G. Tatara, and A. Manchon, Theory of the Topological Spin Hall Effect in Antiferromagnetic Skyrmions: Impact on Current-Induced Motion, Phys. Rev. Lett. 121, 097204 (2018).

[55] S. Lepadatu, BORIS COMPUTATIONAL SPINTRONICS—high performance multi-mesh magnetic and spin transport modelling software, arXiv:2008.02478.

[56] C. Reichhardt and C. J. O. Reichhardt, Noise fluctuations and drive dependence of the skyrmion Hall effect in disordered systems, New J. Phys. 18, 095005 (2016).

[57] C. Reichhardt, D. Ray, and C. J. Olson Reichhardt, Quantized transport for a skyrmion moving on a two-dimensional periodic substrate, Phys. Rev. B 91, 104426 (2015).

[58] J. Müller and A. Rosch, Capturing of a magnetic skyrmion with a hole, Phys. Rev. B 91, 054410 (2015).

[59] J. V. Kim and M. W. Yoo, Current-driven skyrmion dynamics in disordered films, Appl. Phys. Lett. 110, 132404 (2017).

[60] S. A. Díaz, C. J. O. Reichhardt, D. P. Arovas, A. Saxena, and C. Reichhardt, Fluctuations and noise signatures of driven magnetic skyrmions, Phys. Rev. B 96, 085106 (2017).
[61] C. Reichhardt and C. J. O. Reichhardt, Thermal creep and the skyrmion Hall angle in driven skyrmion crystals, J. Phys.: Condens. Matter 31, 07LT01 (2019).

[62] J. M. L. Beaujour, J. H. Lee, A. D. Kent, K. Krycka, and C. C. Kao, Magnetization damping in ultrathin polycrystalline Co films: Evidence for nonlocal effects, Phys. Rev. B 74, 214405 (2006).

[63] J. Bass and W. P. Pratt, Spin-diffusion lengths in metals and alloys, and spin-flipping at metal/metal interfaces: An experimentalist's critical review, J. Phys.: Condens. Matter 19, 183201 (2007).

[64] L. Piraux, S. Dubois, A. Fert, and L. Belliard, The temperature dependence of the perpendicular giant magnetoresistance in $\mathrm{Co} / \mathrm{Cu}$ multilayered nanowires, Eur. Phys. J. B 4, 413 (1998).

[65] S. Zhang, P. M. Levy, and A. Fert, Mechanisms of SpinPolarized Current-Driven Magnetization Switching, Phys. Rev. Lett. 88, 236601 (2002).

[66] C. Petitjean, D. Luc, and X. Waintal, Unified Drift-Diffusion Theory for Transverse Spin Currents in Spin Valves, Domain Walls, and Other Textured Magnets, Phys. Rev. Lett. 109, 117204 (2012). 